\title{
Dresdner Dermatologische Demonstration 2008 - zugleich Tagung der Sächsischen Dermatologischen Gesellschaft am 12. April 2008
}

\author{
Dresden Dermatology Demonstration 2008 - Meeting of the Saxonian Society \\ of Dermatology, April 12, 2008
}

Vorsitz:

Berichterstatter:

Histopathologie:

Klinische Photodokumentation:
Prof. Dr. U. Wollina

Frau Dr. G. Hansel, Dr. A. Koch, Prof. Dr. U. Wollina

Frau Dr. J. Schönlebe, PD Dr. G. Haroske

Frau R. Herz

\section{Bibliografie}

DOI $10.1055 / \mathrm{s}-2007-995775$

Akt Dermatol 2008; 34:

135-142 @ Georg Thieme

Verlag KG Stuttgart · New York ISSN 0340-2541

Korrespondenzadresse

Prof. Dr. Uwe Wollina

Klinik für Dermatologie und

Allergologie am Krankenhaus

Dresden-Friedrichstadt

Städtisches Klinikum

Akademisches Lehrkrankenhaus

der TU Dresden

Friedrichstraße 41

01067 Dresden

wollina-uw@khdf.de
Chronisch vegetierende Pyodermie Azúa mit monoklonaler Gammopathie unklarer Signifikanz

$\nabla$

G. Hansel, U. Schadeberg

Eine 46-jährige Patientin stellt sich mit einem seit Febuar 2007 bestehenden und progredienten Ulcus an der Dorsalseite des rechten Unterschenkels vor. In einem auswärtigen Krankenhaus war die Wunde nach diagnostischer Sicherung als Pyoderma gangraenosum gewertet worden. Eine Lokalbehandlung mit Steroiden und Desinfizenz sowie Kompressionsbandagierung hatten $\mathrm{zu}$ einer deutlichen Besserung des Lokalbefundes geführt. Später war die Wunde abgeheilt. Einige Wochen darauf war ein neues Ulcus direkt über der Ferse entstanden.

Hautbefund: An der Dorsalseite des rechten Unterschenkels fand sich unmittelbar im Bereich der Achillessehne ein lividrotes bis bräunliches papillomatös schwammiges Areal mit Ulzerationen, Fistulationen und entzündlichem Randsaum. Auf Druck entleerte sich stellenweise Pus aus den Fisteln. Proximal davon bestand ein flaches, partiell abgeheiltes fistelnd erodiertes Areal (॰ Abb. 1).

Laborbefunde: CRP 26,4 mg/l, $\alpha_{2}$-Globulin 12,5\%, $\gamma$-Globulin 20,8\%, IgG 16,2 g/l, ASAT 0,82 $\mu \mathrm{kat} / \mathrm{l}$, ALAT $1,22 \mu \mathrm{kat} / \mathrm{l}$, LDH $4,80 \mu \mathrm{kat} / \mathrm{l}$, Albumine $53,1 \%$. In Serum und Urin wurde monoklonales IgG Typ Kappa nachgewiesen.

Mikrobiologische Untersuchung Wundabstrich: Hautnormalflora; Gewebestückchen: kulturell steril; Tb-Kultur vom Gewebestückchen negativ. Mykologische Untersuchung Wundabstrich: mikroskopisch keine Pilzelemente, Kultur steril; Gewebestückchen: Kultur negativ.

Histologie Haut: Mäßige bis schwere granulierende, abszedierende und phlegmonöse Dermatitis mit pseudoepitheliomatöser Epidermishyperplasie.

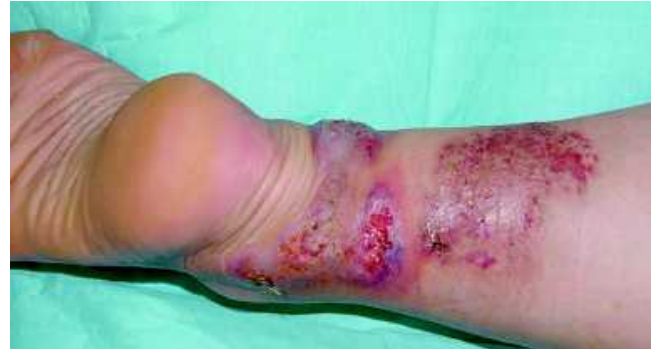

Abb. 1 Chronisch vegetierende Pyodermie Azúa.

Knochenmarkpunktion: Normalbefund.

Bildgebende Diagnostik: Steatosis hepatis, geringgradige Splenomegalie sowie Cholezystolithiasis.

Therapie und Verlauf: Unter konsequenter Feuchtbehandlung der Wunden mit Octenidinhydrochlorid-(Octenisept ${ }^{\circledR}$ )Lösung und Therapie mit 0,7 mg Prednisolon/kg KG kam es zu einer deutlichen Befundbesserung. Die Kortisondosis wurde im weiteren Verlauf langsam auf eine Erhaltungsdosis von $5 \mathrm{mg}$ täglich reduziert. Wegen eines Rezidivs erfolgte die Wiederaufnahme bei fistelndem Wundareal am Malleolus medialis rechts. Erneut besserte sich der Befund unter Lokalbehandlung mit Octenidinhydrochlorid-Lösung. Es erfolgte eine systemische Therapie mit Rifampicin 300 mg $2 \times$ tgl. für 8 Wochen. Darunter stellte sich eine Defektheilung mit postinflammatorischer Hyperpigmentierung ein.

Kommentar: Chronisch vegetierende Pyodermien sind abszedierende Entzündungen unklarer Ätiologie. Es werden 3 Formen unterschieden: der ulzeröse, der verruköse und der papillomatöse Typ. Letzterer wurde 1894 erstmals als chronisch vegetierende Pyodermie von Azúa beschrieben.

Oft, jedoch nicht immer, lassen sich in den Wunden Staphyloccocus aureus, Streptokokken oder Enterokokken nachweisen, deren Rolle in der Pathogenese der Erkrankung jedoch umstritten ist. 
Das Auftreten dieser Pyodermien wird bei Patienten mit Immunsuppression aber auch bei primärer oder sekundärer Immundefizienz beobachtet. Häufig gehen dem Auftreten der Wunden banale Hautinfektionen oder Traumen voraus. Klinisch sehen wir auf lividroten Infiltraten Pustulationen, kleinere und größere Nekrosen und Ulzerationen, Unterminierungen, Fisteln und Gänge sowie verruziforme und papillomatöse Vegetationen [1]. Im histologischen Befund imponieren eine pseudoepitheliomatöse Hyperplasie und dermale, phlegmonöse Abszesse. Je nach mikrobiologischem Untersuchungsbefund sollte eine gezielte antibiotische Therapie mit Kortikosteroiden oder/und anderen Immunsuppressiva kombiniert werden. Eine alleinige Antibiose führt in der Regel nicht zur Abheilung. Für die lokale Behandlung haben sich desinfizierende Lösungen (feuchte Umschläge) bewährt. Eine chirurgische Abtragung der Vegetationen ist möglich, jedoch nicht immer erfolgreich. Der Einsatz von TNF-alphaHemmern kann im Einzelfall erwogen werden [2]. Differenzialdiagnostisch ist an tiefe Mykosen, Aktinomykose, Nokardiosen zu denken [3]. Die klare Abgrenzung zum Pyoderma gangraenosum ist schwierig und im Einzelfall nicht immer möglich.

\section{Literatur}

1 Armbruster M, Schimmer M, Sander CA. Chronisch vegetierende Pyodermie Typ Azúa bei Plasmozytom Typ IgA/kappa. J Dtsch Derm Ges 2007; 5: 89

2 Carrera C, Mascaró JM Jr, Moreno-Romero JA, Iranzo P, Palou J, Zamora $E$, Herrero $C$. Pyoderma vegetans associated with severe psoriatic arthritis: good response to etanercept. Dermatology 2007; 214: 77-81

3 Dissemond J, Körber A, Grabbe S. Differentialdiagnosen des Ulcus cruris. J Dtsch Derm Ges 2006; 4: 627-634

\section{Dermatomyositis mit nur passagerer Myositis und sekundärer Amyloidose $\nabla$ \\ K. Hasenöhrl, G. Rahmig, C. Herrman, J. Schönlebe}

Seit 16 Jahren leidet die 36-jährige Patientin unter rötlichen Flecken der Haut, welche bisher als Morphea eingeordnet wurden. Zusätzlich klagt sie über Missempfindungen und Schwäche der Muskulatur der Extremitäten. Eine Verschlechterung durch Sonne wird verneint. Eine Raynaud-Symptomatik besteht nicht.

Hautbefund: Es fanden sich an den Streckseiten der Oberarme und an den Oberschenkeln braunrote, konfluierende Erytheme, teilweise auch diskrete Infiltrate ( $\bullet$ Abb.2). An den Fingerrücken der rechten Hand konnte man angedeutet Gottronsche Papeln erkennen. Im Gesicht bestand ein diffuses Erythem, welches sich diskret auch auf die Kopfhaut fortsetzte.

Laborbefunde: Leukozyten 25/ $\mu$ l, ANA: positiv 1:320, SmoothMuscle-AK positiv 1:320.

Histologie (Oberschenkel rechts): Nachweis kutaner, schwach Zytokeratin 5/6-positiver Amyloidablagerungen im Papillarkörper entsprechend einer atypischen kutanen Amyloidose.

Bildgebende Diagnostik: unauffällig.

EKG: Normalbefund.

Therapie und Verlauf: Hydroxychloroquin (Quensyl ${ }^{\circledR}$ ) $200 \mathrm{mg}$ tgl. Darunter Abklingen der Muskelschwäche, jedoch bislang keine Regression der Hautveränderungen.

Kommentar: Die Dermatomyositis ist mit einer Inzidenz von < 1: 100000 eine relativ seltene Haut-Rheuma-Erkrankung. Eine Beteiligung von Virusinfektionen wird diskutiert. Die Autoimmunpathogenese wird durch das Vorhandensein von spezifischen AK und der kutanen Immunpathologie belegt [1, 2].

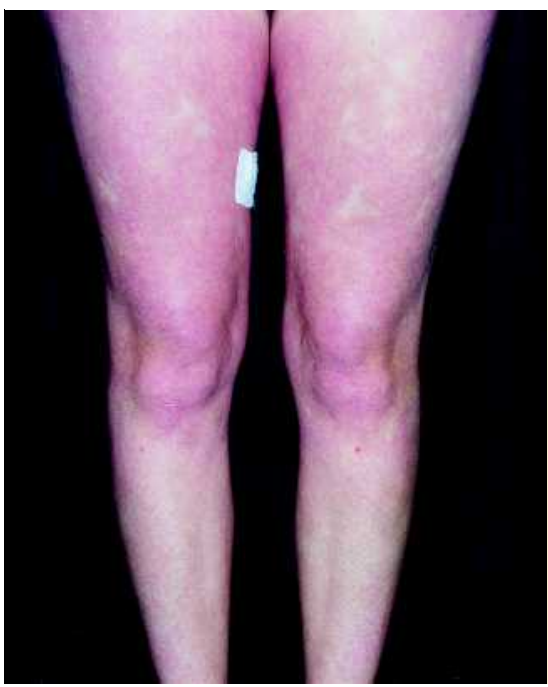

Abb. 2 Amyotrope Dermatomyositis.

Obwohl die Kombination von Dermatitis und Myositis namensgebend und charakteristisch für die Erkrankung ist, existiert auch eine amyopathische Verlaufsform ähnlich wie bei unserer Patientin im weiteren Verlauf.

Bemerkenswert ist, dass sich bei dieser milderen Verlaufsform eine sekundäre Amyloidose manifestiert hat. In der internationalen Literatur sind in den letzten Jahrzehnten nur sporadisch derartige Konstellationen beschrieben worden [3]. Die Dunkelziffer ist möglicherweise höher. Als Konsequenz empfehlen wir die Suche nach einer kutanen Amyloidose auch dann, wenn es sich klinisch eindeutig um eine rein amyotrophe Dermatomyositis handelt.

Aufgrund der alleinigen kutanen Beteiligung unserer Patientin ohne Myositis entschieden wir uns für die Behandlung mit Hydroxychloroquin als Basistherapeutikum.

\section{Literatur}

1 Dourmishev LA, Wollina U, Hipler U-C, Peytcheva V. Myositis-specific and myositis-associated autoantibodies in dermatomyositis and polymyositis. Clin Appl Immunol 2006; 5: 553 - 557

2 Dourmishev LA, Wollina U. Dermatomyositis: immunopathologic study of skin lesions. Acta Dermatovenereol APA 2006; 15: 45-51

3 Horiguchi Y, Mitani T, Danno K, Ozaki M, Fine JD, Leigh IM, Imamura S. Extensive variant of cutaneous amyloidosis: report of a case with electron-microscopic and immunohistochemical studies of the basement membrane zone at sites of amyloid production. Dermatology 1992; 185: $181-189$.

\section{Ulzerationen der Füße als Erstmanifestation eines Diabetes mellitus \\ $\nabla$ \\ D. Langner, U. Schadeberg}

Seit Ende Dezember 2007 bemerkte der 53-jährige Patient Blasen an den Großzehenballen, aus denen sich schmierig belegte, flache Ulzerationen mit einem sekundären Erysipel der Unterschenkel entwickelten. Eine Psoriasis vulgaris war bekannt.

Hautbefund: Es zeigten sich an den Großzehenballen und der Plantarseite der Großzehen beidseits flache, schmierig belegte Ulzerationen (re $7 \times 6 \mathrm{~cm}$, li $9 \times 7 \mathrm{~cm}$ ) mit randständigen Blasenresten. Die Unterschenkel und die Füße beidseits waren diffus gerötet, geschwollen und überwärmt. Nebenbefundlich waren an den unteren Extremitäten einzelne flache, infiltrierte, nummuläre Psoriasisherde auffällig ( $\bullet$ Abb. 3). 


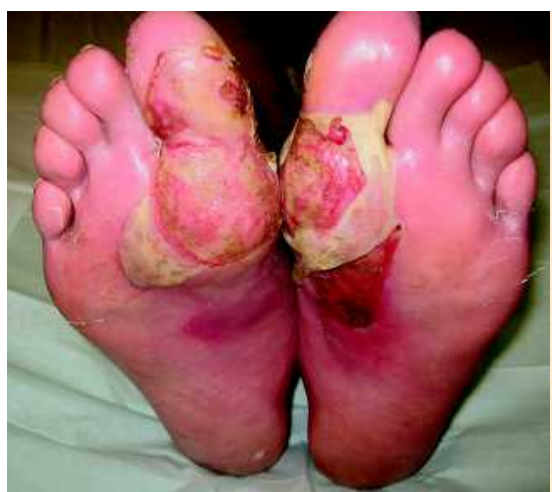

Abb. 3 Diabetisches Fußsyndrom, initial unter dem Bild einer Blasenbildung.

Laborbefunde: CRP $145.4 \mathrm{mg} / \mathrm{l}$, Leukozyten $13.07 \mathrm{Gpt} / \mathrm{l}$, Thrombozyten $355 \mathrm{Gpt} / \mathrm{l}$, LDH $4.69 \mu \mathrm{kat} / \mathrm{l}$, Glukose im Serum 26.0 mmol/l, HbA1c $13.0 \%$.

Mykologie Abstrich vom Zehenzwischenraum: Candida parapsilosis sehr viel, Trichosporon cutaneum sehr viel.

Mikrobiologie Abstrich vom Ulcus: Acinetobacter baumanii/calcoaceticus: mäßig viel, Enterobacter cloacae und Staphylococcus aureus: viel, $\beta$-hämolysierende Streptokokken - Mengeneinschätzung nicht möglich, Hautnormalflora: viel. Resistenz sensibel ggü. Ampicillin/ Sulbactam.

Bildgebende Diagnostik: Erhebliche Großzehengrundgelenksarthrose beidseits, rechts ausgeprägter als links mit deutlicher Weichteilschwellung in diesem Bereich, sonst unauffällig.

ENG und EMG: Elektrophysiologisch deutlich schwere demyelinisierende Polyneuropathie.

Augenärztliches Konsil: Ophthalmologischer Normalbefund.

Therapie und Verlauf: Es wurde eine systemische Antibiose mit Ampicillin/Sulbactam (Unacid ${ }^{\circledR}$ ) über 7 Tage vorgenommen. Nach initialer lokaler Wundbehandlung mit Octenisept ${ }^{\circledR}$-Feuchtumschlägen wechselten wir im Verlauf auf Pasta argenti nitrici $1 \%$ Standardrezeptor (SR) und führten eine Kompressionsbandagierung durch. Auf Empfehlung des Neurologen erfolgte aufgrund der Polyneuropathie eine intravenöse Therapie mit Thioctacid 600 über $14 \mathrm{~d}$, welche bei Entlassung auf orale Gabe umgestellt wurde. Unter engmaschigen Blutzuckerkontrollen erfolgte eine Insulineinstellung, sodass bei Entlassung eine normoglykämische Stoffwechsellage bestand. Innerhalb von 3 Wochen heilten die Ulzerationen komplett ab.

Kommentar: Die ätiologisch entscheidenden Faktoren für die Entwicklung der diabetischen Fußulzera sind die diabetische Polyneuropathie und die Makroangiopathie. Die klassische diabetische sensomotorische Polyneuropathie beginnt bevorzugt distal an den unteren Extremitäten und ist in der Regel symmetrisch ausgeprägt. Die motorische Innervationsstörung führt zu einer Muskelatrophie und Verkürzung der Fußbinnenmuskulatur (intrinsische Muskulatur) mit Ausbildung von Hammerbzw. Krallenzehen.

Dermatologische Symptome können auf einen bisher unerkannten Diabetes mellitus hinweisen, wie in unserem Fall [1]. Deshalb ist es unverständlich, dass im Disease Management Programme Diabetes eine regelhafte dermatologische Beteiligung nicht vorgesehen ist. Dies ist nachteilig für Patienten und untergräbt Möglichkeiten der Sekundärprophylaxe.

\section{Literatur}

1 Köstler E, Porst H, Wollina U. Cutaneous manifestations of metabolic diseases: uncommon presentations. Clin Dermatol 2005; 23: $457-464$

\section{Keratosis palmoplantaris transgrediens} et progrediens (Greither)

\section{Krönert, J. Heinze}

Bei dem 5-jährigen Jungen bestehen seit dem Säuglingsalter progrediente ekzemartige Hautveränderungen an beiden Händen. Zudem fällt eine deutliche palmoplantare Hyperhidrose auf. In der Familie traten bisher keine vergleichbaren Hautveränderungen auf.

Hautbefund: An Handflächen und Fußsohlen finden sich rötlichgelbliche Keratosen mit diskreter Schuppung mit einem Übergreifen auf Handgelenk und Fingerstreckseiten, jedoch ohne Befall von Ellenbogen, Knöchel oder Knie ( $\bullet$ Abb. 4). Im Erlanger Atopie-Score nach Diepgen werden 4 Punkte erreicht.

Laborbefunde: Mykologisches Nativpräparat und Kultur negativ. Therapie: Lokalbehandlung mit keratolytischen (harnstoff- und salicylsäurehaltigen) und pflegenden Externa, beispielsweise 5-10\%-ige Salicylvaseline und Ungt. acidi lactici 0,4\% WILSR.

Kommentar: Die hereditären Palmoplantarkeratosen (PPK) stehen den differentialdiagnostisch $\mathrm{zu}$ trennenden symptomatischen Formen (infektiös, toxisch, endokrinologisch) gegenüber. Im vorliegenden Fall handelt es sich nach klinischem Befund und Anamnese am ehesten um die 1952 erstmals von Greither beschriebene Keratosis extremitarum hereditaria transgrediens et progrediens dominans [2]. Ursächlich liegt eine Mutation im Keratin 1 zugrunde [1]. Diese seltene PPK manifestiert sich vorwiegend im ersten Lebensjahrzehnt mit dem symmetrischen Auftreten von papulösen bis flächenhaften, weißgrauen Keratosen mit lividrotem Rand palmoplantar, die im Verlauf auf Handund Fußrücken, Fersen, Knie und Ellenbogen übergreifen können. Typisch ist eine begleitende Hyperhidrose. Die Progredienz sistiert meist in der 4. Dekade und es schließt sich eine Rückbildung der Erkrankung an. Die Symptomausprägung innerhalb der Familie kann sehr variabel sein [1,3].

Die Therapie erfolgt symptomatisch keratolytisch mit Salicylsäure-, Urea- oder Vitamin-A-Säure-haltigen Externa, durch mechanische Abtragung und bei ausgeprägten Formen ggf. mit oralen Retinoiden. Allerdings sind die Therapieerfolge mit Retinoiden leider meist nicht überzeugend, da die sich einstellende Vulnerabilität der Haut größere Probleme als die Hyperkeratosen verursachen kann.

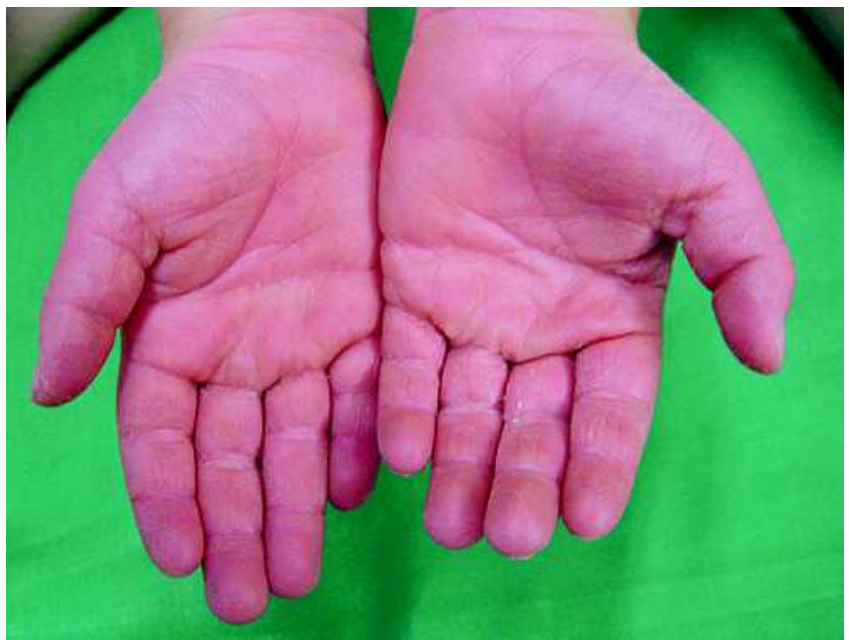

Abb. 4 Morbus Greither: Flächiges Palmarerythem mit geringgradiger Hautverdickung, transgrediente Ausdehnung. 


\section{Literatur}

1 Gach JE, Munro CS, Lane EB, Wilson NJ, Moss C. Two families with Greither's syndrome caused by a keratin 1 mutation. J Am Acad Dermatol 2005; 53: $225-230$

2 Greither A. Keratosis extremitarum hereditaria transgrediens et progrediens mit dominatem Erbgang. Hautarzt 1952; 3: 198-203

3 Wollina U, Knopf B, Schaaschmidt H, Frille I. Familiäre Koexistenz von Erythrokeratodermia variabilis und Keratosis palmoplantaris transgrediens et progrediens. Hautarzt 1989; 40: 169-172

\section{Polyzyklische Erytheme bei Mycosis fungoides unter Bexarotentherapie \\ $\nabla$}

A. Koch, D. von Jagow

Seit dem Frühjahr 2001 besteht bei der 76-jährigen Patientin eine Mycosis fungoides (Mf) im Patchstadium. In den folgenden drei Jahren konnte durch Photochemotherapie (Bade- \& CremePUVA) und topische Glukokortikoide ein stabiler Befund erhalten werden. Im Jahr 2005 entwickelten sich zusehends stärker infiltrative Plaques. Wir begannen eine systemische Behandlung mit Bexaroten (Targretin ${ }^{\circledR}$ ), die jedoch von der Patientin nicht fortgeführt wurde. Daraufhin setzten wir bei Progress der Erkrankung Methotrexat in Dosen von 10-25 mg pro Woche ein. In den folgenden Monaten waren neben der Entwicklung von kutanen Tumoren auch ein Organbefall durch das Lymphom verdächtigt worden. So erfolgte im Juli 2006 eine partielle Parotidektomie links. Histologisch wurden Infiltrate der bekannten Mf vermutet. Intermittierend wurden einige Tumoren mittels Weichstrahlröntgentherapie erfolgreich behandelt. Bei Verschlechterung des Lokalbefundes und in Absprache mit der Patientin entschlossen wir uns im Mai 2007 erneut zur Einstellung auf Bexaroten, wobei nach gutem Ansprechen eine Dosisreduktion auf 2-3 Kapseln pro Tag durchgeführt werden konnte. Nach einer psychischen Stresssituation im Oktober 2007 entwickelten sich ausgedehnte erythematöse, teils gyrierte Herde am gesamten Integument bei begleitender Minderung des Allgemeinzustandes.

Hautbefund: Es fanden sich oberflächlich erodierte Plaques auf dem Rücken und an der rechten Flanke sowie weniger stark infiltrative Plaques und Maculae am übrigen Integument mit rötlichen urtikariellen gyrierten Herden bis $20 \mathrm{~cm}$ Größe ( $\odot$ Abb. 5 a). Teilweise zeigten sich die Hautveränderungen nässend. Tumoröse Efflereszenzen konnten nicht nachgewiesen werden. Zervikal links fielen narbige Residuen mit Erythemen und atrophen Arealen auf.

Laborbefunde: Löslicher Interleukin-2-Rezeptor $5074 \mathrm{U} / \mathrm{ml}$, Hypalbuminämie $45,9 \%$.

Histologie (aus polyzyklischem Erythem am Abdomen): Mitteldichtes oberflächlich perivaskulär lokalisiertes, betont lymphomonozytoides-histiozytäres Infiltrat mit Beimengungen von Mastzellen und zahlreichen eosinophilen Granulozyten. Ausgewogenes Verhältnis von CD4- zu CD8-positiven Lymphozyten. Am ehesten reaktiv entzündliche Veränderungen, keine Lymphominfiltrate $(\bullet$ Abb.5b).

Bildgebende Diagnostik: Harnstauungsniere links, Leberhämangiom. Inguinal multiple vergrößerte Lymphknoten bis $19 \mathrm{~mm}$, Struktur weitestgehend erhalten. Axillär kein Nachweis auffälliger Lymphknoten.

Therapie und Verlauf: Unter lokaler Therapie mit BetamethasonV-Creme 0,1\% NRF und begleitender UVB 311-nm-Bestrahlung kam es innerhalb weniger Tage zur Rückbildung der generali-

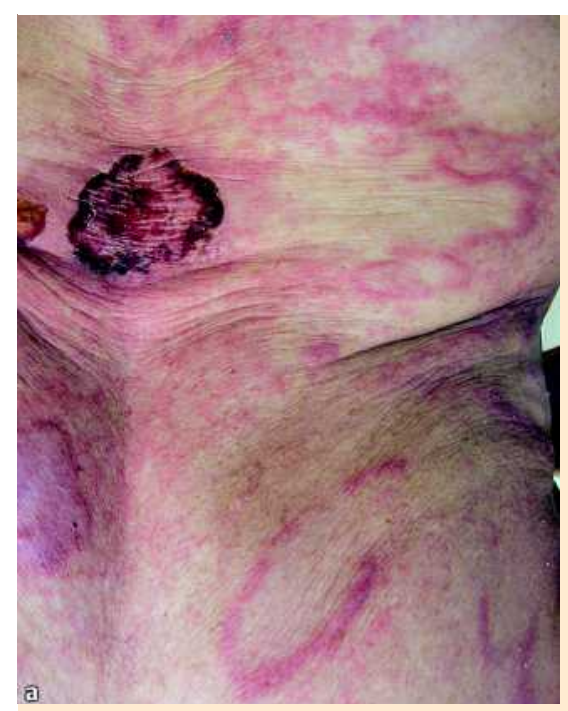

Abb. 5 a Polyzyklische Erytheme unter Bexaroten-Therapie. b Histologie zu Abb. 5 a: Unspezifisches perivaskuläres Infiltrat. $\mathrm{HE}, \times 10$.

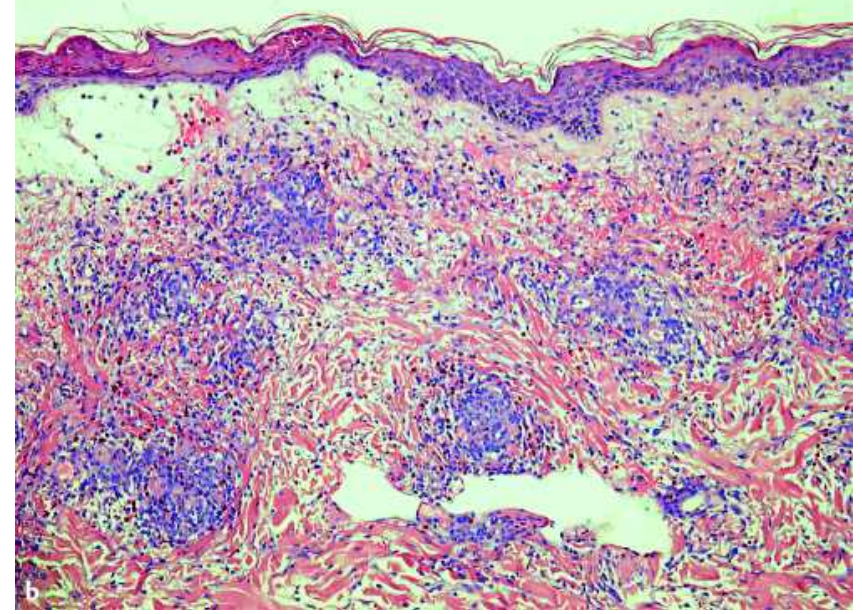

sierten Hauterscheinungen. Zurück blieben lediglich die typischen Effloreszenzen der bekannten Mycosis fungoides.

Kommentar: Das Rexinoid Targretin ${ }^{\circledR}$ (Bexaroten) ist ein wirksames Therapeutikum bei Mf. Als klassische Nebenwirkungen sind eine Hypertriglyceridämie, Hypothyreose und Abgeschlagenheit zu verzeichnen [3]. Die Stoffwechselveränderungen werden mit Fenofibrat und L-Thyroxin behandelt [1].

Eine seltenere Nebenwirkung stellt das akut einsetzende Erythem dar [2, 3]. Wie in unserem Fall entwickeln sich rasch aufschießende Erytheme und Infiltrate, die histologisch kein spezifisches Substrat aufweisen. Möglicherweise ist diese Nebenwirkung mit einem guten Ansprechen der Tumore auf Bexaroten assoziiert [2]. Die Therapie mit Bexaroten kann fortgeführt werden.

\section{Literatur}

1 Assaf C, Bagot M, Dummer R, Duvic M, Gniadecki R, Knobler R, Ranki A, Schwandt P, Whittacker S. Minimizing adverse side-effects of oral bexarotene in cutaneous T-cell lymphoma: an expert opinion. Br J Dermatol 2006; 155: $261-266$

2 Govindan R, Crowley J, Schwartzberg L, Kennedy P, Williams C, Ekstrand $B$, Sandler A, Jaunakais D, Bolejack V, Ghalie R. Phase II trial of bexarotene capsules in patients with advanced non-small-cell lung cancer after failure of two or more previous therapies. J Clin Oncol 2006; 24: $4848-4854$

3 Lowe MN, Plosker GL. Bexarotene. Am J Clin Dermatol 2000; 1 : $245-250$ 


\section{Lupus erythematodes tumidus}

$\nabla$

\section{J. Runge, U. Gabsch}

Eine 34-jährige Patientin stellte sich mit rezidivierenden juckenden, ekzematoiden Hautveränderungen im Gesicht und am Dekolleté vor, weil sich die Abstände der Schübe verkürzten. Der Patientin ist eine mögliche Provokation durch Sonne aufgefallen. Im Jahr 2006 kamen streifige Hautveränderungen entlang der Axillen hinzu.

Hautbefund: Es fanden sich disseminiert an Wange, Kinn und Hals rötlich-livide, makulopapulöse, girlandenartig aufgereihte Effloreszenzen ( $\bullet$ Abb.6a). Einzelne, diskrete Papeln waren auch an Oberarmen und Rücken zu finden.

Laborbefunde: ANA positiv 1:80, Differenzierung negativ.

Histologie: Epidermis unauffällig mittelbreit mit orthokeratotischer Verhornung. Keine nennenswerten hydropischen Basalzelldegenerationen. Epidermale Basalmembran zart. In allen Dermisetagen ein teils perivaskulär, teils periadnexiell lokalisiertes, (fleckförmig) dichtes, von Lymphozyten dominiertes entzündliches Infiltrat mit nur wenigen beigemischten monozytoiden Zellen, Mastzellen und einzelnen neutrophilen Granulozyten. Recht ausgeprägte Muzinablagerungen in der retikulären Dermis ( $\bullet$ Abb.6b). Direkte Immunfluoreszenz: unspezifische Fibrinogenreaktion interstitiell intradermal.

Lichttreppe für UVA/UVB: Erhöhte UVB-Lichtempfindlichkeit.

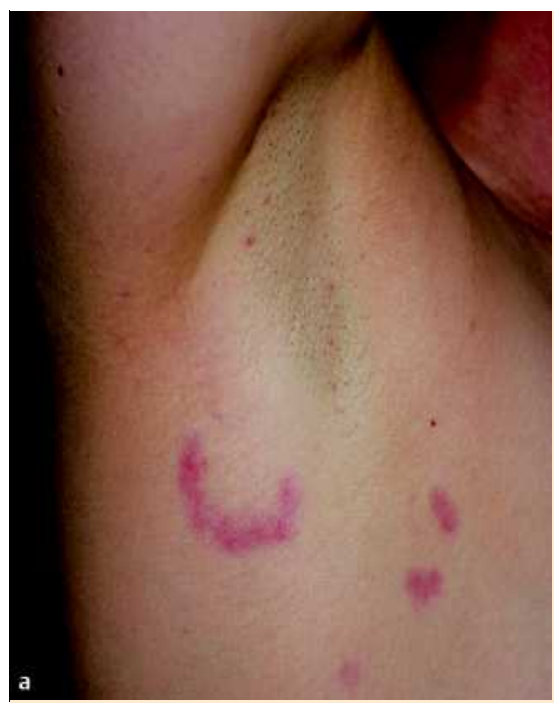

Abb. 6 a Klinisches Bild des Lupus tumidus. b Histologie zu Abb. 6 a: Muzinfärbung, $\times 20$.

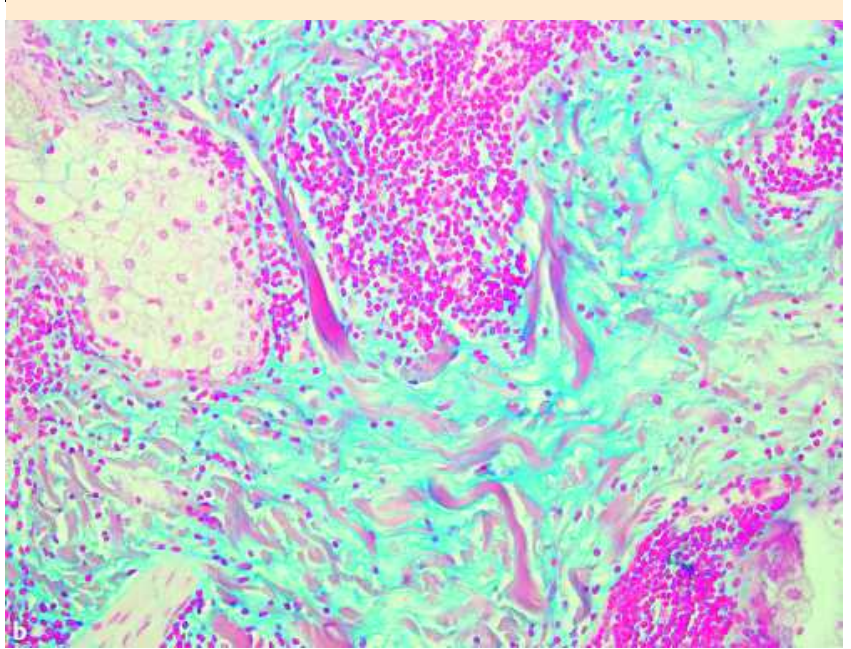

Bildgebende Diagnostik: unauffällig.

Therapie und Verlauf: Therapie mit Hydroxychloroquin (Quensyl ${ }^{\circledR}$ ) $200 \mathrm{mg} / \mathrm{d}$ intern, Betamethason-V-Creme 0,1\% NRF für den Körper und Prednicarbat (Dermatopcreme ${ }^{\circledR}$ ) anfänglich für 2 Tage für das Gesicht.

Kommentar: Der Lupus erythematodes tumidus (LET) wird als eine Sonderform des chronisch kutanen Lupus erythematodes angesehen. Das Erkrankungsalter liegt zwischen dem 20. und 40. Lebensjahr, Frauen sind häufiger betroffen als Männer [2,3]. Er ist gekennzeichnet durch ein entzündliches Infiltrat in der Dermis und typischen geröteten, urtikariaähnlichen, über dem Hautniveau liegenden Herden. Prädelektionsstellen sind die lichtexponierten Areale wie Gesicht, Dekolleté und Arme [2 -4]. Der LET ist in 75\% durch UVA/UVB-Strahlung provozierbar, dies erklärt auch die verstärkte Symptomatik in den Sommermonaten mit häufigen Rezidiven [1]. Therapeutisch ist eine systemische Therapie mit Antimalariamitteln effektiv. Unterstützend kommen lokale Kortikoide zur Anwendung [2, 3]. Alternativ stehen topische Calcineurininhibitoren zur Verfügung [5]. Die Aufklärung des Patienten über den notwenigen Lichtschutz mit UVA/UVB-Filter ist erforderlich.

\section{Literatur}

1 Kuhn A, Sonntag M, Richter-Hintz D, Oslislo C, Megahed M, Ruzicka T, Lehmann P. Phototesting in lupus erythematosus tumidus - review of 60 patients. Photochem Photobiol 2001; 73: 532-536

2 Kuhn A, Richter-Hintz D, Oslislo C, Ruzicka T, Megahed M, Lehmann P. Lupus erythematosus tumidus - a neglected subset of cutaneous lupus erythematosus: report of 40 cases. Arch Dermatol 2000; 136: $1033-1041$

3 Vieira V, Del Pozo J, Yebra-Pementel MT, Martínez W, Fonseca E. Lupus erythematosus tumidus: a series of 26 cases. Int J Dermatol 2006; 45 : $512-517$

4 Wollina $U$, Hansel G. The use of topical calcineurin inhibitors in lupus erythematosus: an overview. J Eur Acad Dermatol Venereol 2008; 22: $1-6$

1 Wollina U, Hein G. Lupus erythematoses - uncommon presentations. Clin Dermatol 2005; 23: 470-479

\section{Foudroyant verlaufende pansklerotische Morphaea des Erwachsenenalters $\nabla$ \\ B. Heinig, U. Gabsch}

Anamnese: Der 69-jährige Patient wurde wegen rezidivierender Tachykardien mit Vorhof-Flimmern und nach Kardioversion mit Questimed-(Clexane $\left.{ }^{\circledR}\right)$ Injektionen behandelt. Wenig später zeigten sich erstmals sklerodermiforme Hautveränderungen. Es wurde die Umstellung auf Phenprocoumon (Falithrom ${ }^{\circledR}$ ) vorgenommen. Im Sommer 2007 wurde ein Rektumkarzinom (Mukosakarzinom Wien 4.1) endoskopisch entfernt. Im November 2007 erfolgte die stationäre Einweisung wegen zunehmender Hautsklerosierung mit thorakalem Engegefühl.

Hautbefund: Bei Aufnahme bot nahezu das gesamte Integument eine derbe Sklerose mit livid-entzündlicher Randzonenreaktion. Atemexkursionen waren wegen beginnender Einmauerung des Thorax kaum sichtbar. Die Extremitäten erschienen eingeschnürt und dadurch teilweise bewegungseingeschränkt (๑ Abb. 7).

Laborbefunde: CRP 11,4 mg/l, Antistreptolysin 222,0 U/ml, Quickwert 51.5\% (unter Falithrom ${ }^{\circledR}$ ), Alpha-1-Globulin 3,5\%, Alpha-2-Globulin 12,4\%. Laborchemisch kein Nachweis spezifischer Antikörper. 


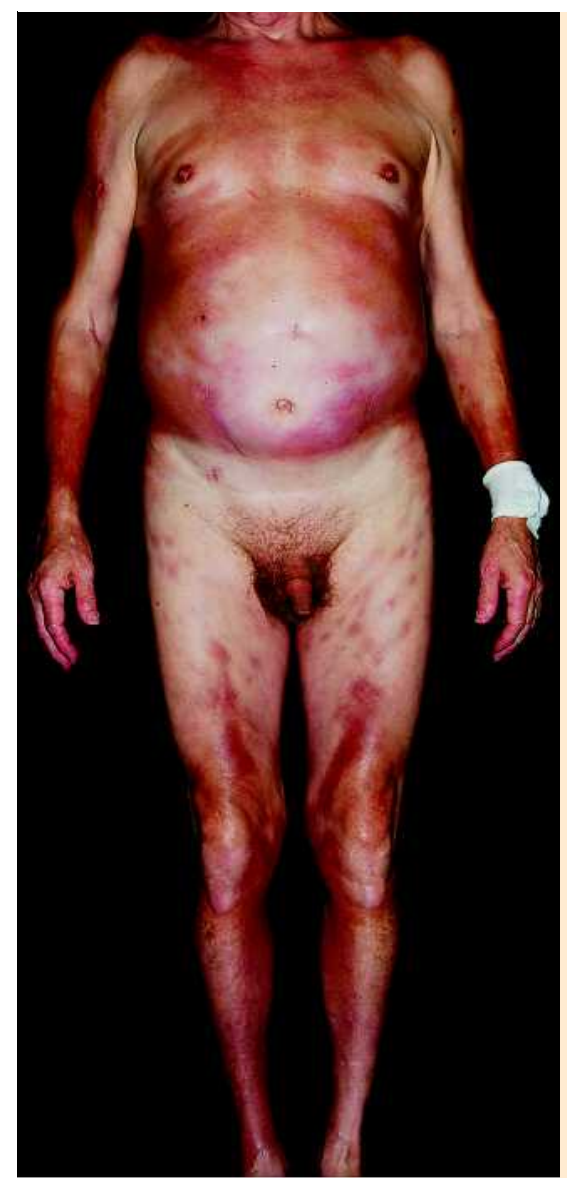

Abb. 7 Pansklerotische Morphaea.

Histologie: Hautexzisate von Rumpf und Oberarm ergaben die Befundkombinationen einer sklerosierenden Dermatitis, vereinbar mit einer Sklerodermie/Morphaea. Eine zusätzlich veranlasste Borrelien-PCR aus dem Gewebe erbrachte keine Auffälligkeiten.

Bildgebende Diagnostik: Gering verzögerte Passage des Reisbreibolus durch den Ösophagus.

Bodyplethysmographie: Keine Ventilationsstörungen, Säure-Basen-Haushalt ausgeglichen, Normoxämie.

Diffusion Single-Breath: Keine Diffusionsstörungen, keine Verteilungsstörungen.

Echokardiographie: Normale Aorten-Vorhof-Relation ohne Hinweis auf Vorhofdilatation. Linker Ventrikel beginnend konzentrisch hypertrophiert, nicht dilatiert. Kein Hinweis auf eine diastolische Funktionsstörung. Rechter Ventrikel nicht hypertrophiert oder dilatiert. Normale Kinetik des LV, EF ca. 60\%. Zarte Mitral-, Aorten-, Pulmonal- und Trikuspidalklappe mit MI 1(2), minimaler AI und PI sowie TI 1(2). Keine pulmonale Hypertonie (pRVsys um $29 \mathrm{mmHg}$ ), kein Perikarderguss.

Therapie und Verlauf: In Anbetracht des rasch progredienten Verlaufes kam neben einem Prednisolonkurzstoß mit $50 \mathrm{mg}$ tgl. MTX mit $25 \mathrm{mg}$ pro Woche zum Einsatz. Als antifibrotische Therapie wurden Penicillin-G-Infusionen (zweimal tgl. 5 Mio. IE) über 11 Tage appliziert. Die Hautpflege erfolgte mit Ungt. acidi lact. 0,4\% W/L SR. Darüber hinaus erhielt der Patient zweimal täglich intensive physiotherapeutische Anwendungen mit Atemtherapie, manueller Lymphdrainage, klassischer und Bindegewebsmassage, Gelenkmobilisation einschließlich der Rippengelenke, Dehnungslagerungen und Kinesitherapie im PNFMuster (propriozeptive neuromuskuläre Fazilitation). Unter die- ser Komplextherapie besserte sich die Sklerose subjektiv und objektiv deutlich. Hautfalten wurden wieder abhebbar. Die thorakale Atemexkursion Exspiration/Inspiration betrug bei Entlassung $4 \mathrm{~cm}$ über den unteren Aperturen.

Kommentar: Die Sklerodermie ist eine autoimmune Erkrankung mit lederartiger Verhärtung und Sklerose der Haut. Zwei Hauptformen werden unterschieden: die progressiv-systemische Sklerodermie PSS sowie die zirkumskripte Sklerodermie (Morphaea) mit lokalisierten Plaqueformationen ohne Beteiligung viszeraler Organe [2]. Die Morphaea (M) kann wie folgt klassifiziert werden: Plaquemorphaea, generalisierte $M$, bullöse $M$, lineare $M$ und tiefe M [1]. Spezifische Autoantikörper wie bei systemischer Sklerose (Centromer oder SCL-70) lassen sich bei der M in der Regel nicht nachweisen. Antinukleäre Antikörper sind möglich, jedoch mit niedrigerem Titer als bei systemischer Sklerose [1, 4]. Borrelieninfektionen scheinen für die Auslösung der M keine Rolle zu spielen [2].

Als seltene Variante der M involviert die Pansklerose alle Schichten der Haut und beteiligt subkutanes Gewebe bis hin zu Muskel-, Sehnen- und Knochenstrukturen, woraus nicht selten Gelenkkontrakturen mit schmerzhaften Bewegungseinschränkungen resultieren. Während die PSS eher eine Erkrankung des mittleren Lebensalters ist, wird über - auch schwer verlaufende Formen der $\mathrm{M}$ bereits im Jugendalter berichtet [5].

Zur topischen Anwendung kommen Kortikosteroide und Calcineurinhemmer. Systemisch können antifibrotisch Penicillin und immunsuppressiv Kortikosteroide, Methotrexat oder andere Immunsuppressiva (z. B. Azathioprin, Cyclosporin A) erwogen werden. UVA/UVA1 und PUVA sind im Einzelfall wirksam [5]. Obligat ist ab Diagnosestellung die Einbeziehung gezielter physiotherapeutischer Maßnahmen mit antifibrotischer/antiödematöser, hyperämisierender, mobilisierender/funktionserhaltender und analgetischer Zielstellung in das Therapiekonzept [3]. Die Patienten sollten psychologisch geführt werden.

\section{Literatur}

1 Bielsa I, Ariza A. Deep morphea. Semin Cutan Med Surg 2007; 26-95

2 Goodland JR, Davidson MM, Gordon P, Billigton R, Ho-Yen DO. Morphea and Borrelia burgdorferi: results from the Scottish Highlands in the context of the world literature. Mol Pathol 2002; 55: 374-378

3 Knopf B, Barta U, Uhlemann C, Wollina U. Physiotherapeutische Möglichkeiten bei Kollagenosen. Dermatol Monatsschr 1989; 175: $240-247$

4 Maragh SH, Davis MDP, Bruce AJ, Nelson AM. Disabling pansclerotic morphea: Clinical presentation in two adults. J Am Acad Dermatol 2005; 53: S115-S119

5 Wollina U, Buslau M, Heinig B, Petrov I, Unger L, Kyriopoulou J, Koch A, Köstler E, Schönlebe J, Haroske G, Doede T, Pramatarov K. Disabling pansclerotic morphea of childhood poses a high risk of chronic ulceration of the skin and squamous cell carcinoma. Int J Low Extrem Wounds 2007; 6: $291-298$

\section{Pemphigus vegetans (Hallopeau) der Kopfhaut unter dem klinischen Bild einer Tinea capitis} $\nabla$

\section{A. Gemmeke, B. Eichinger}

Seit ca. einem Jahr leidet der 47-jährige Patient an entzündlichen, nässenden, verkrusteten Hautveränderungen der Kopfhaut mit büschelartigem Haarausfall. Eine ambulant durchgeführte externe wie interne Kortisonbehandlung habe eine kurzfristige Besserung mit promptem Rezidiv nach Therapieende erbracht. 


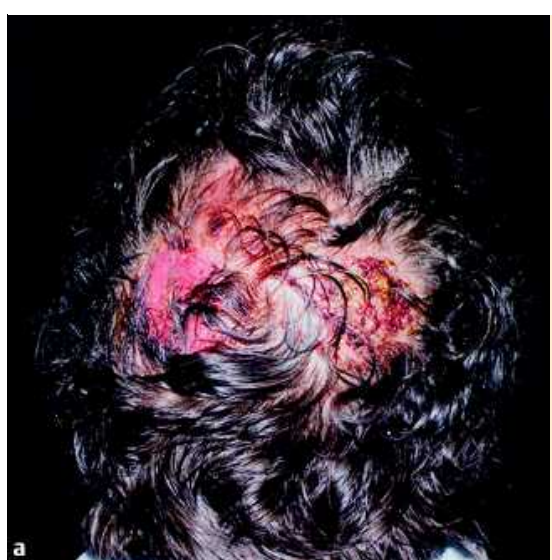

Abb. 8 a Pemphigus vegetans der Kopfhaut. b Akantholytische Spaltbildung. $\mathrm{HE}, \times 20$.

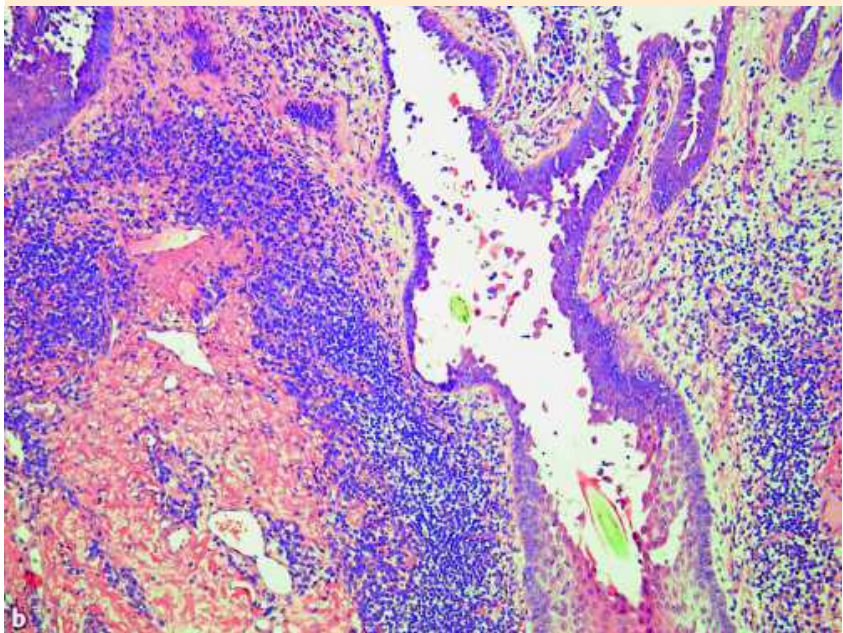

Hautbefund: An der Kopfhaut fanden sich mehrere fast kreisrunde, bis $8 \mathrm{~cm}$ im Durchmesser große, entzündlich-verkrustete Herde $(\bullet$ Abb. 8 a). Die Haare waren leicht epilierbar.

Laborbefunde: Pemphigus-Antikörper 1:640.

Histologie: Chronisch-fortdauernde oberflächlich perivaskuläre sowie interstitielle und periadnexielle Dermatitis mit ausgeprägten Akantholysen ( $\mathbf{A b b} \mathbf{8} \mathbf{8}$ b). Histologisch kein Pilznachweis. In der direkten Immunfluoreszenz herdförmig granuläre Immunreaktion für IgG und C3.

Mykologische Befunde: Epilierte Kopfhaare: mikroskopisch sehr viele Sprosszellen. Pilzkultur von Haaren und Abstrich negativ.

Therapie und Verlauf: Aufgrund des klinischen Befundes und des mikroskopischen Nachweises von Sprosszellen bei epilierten Kopfhaaren war zunächst an eine Trichophytie gedacht worden, sodass initial eine kombinierte antimykotische Therapie mit Itrakonazol (Sempera ${ }^{\circledR}$ 100) und Ciclopirox-(Batrafen ${ }^{\circledR}$ ) Creme eingeleitet wurde. Nach definitiver Diagnosestellung interne Therapie mit initial $100 \mathrm{mg} / \mathrm{d}$ Prednisolon und $150 \mathrm{mg} / \mathrm{d}$ Azathioprin. Unter dieser Behandlung konnte sowohl eine Remission der Hautveränderungen als auch neues Haarwachstum beobachtet werden.

Kommentar: Der Pemphigus vegetans stellt eine seltene Variante des Pemphigus vulgaris dar. Sein Anteil an der Gesamtheit der Pemphiguserkrankungen beträgt 1-2\%. Neben Erosionen und Blasen entwickeln die Patienten chronisch-vegetierende, verrukös-granulomatöse Plaques. Historisch werden der Typ Neumann, der zumeist einen ausgedehnten, therapierefraktären Verlauf nimmt, und der Typ Hallopeau, eine lokalisierte Variante, unterschieden [1]. Bevorzugte Lokalisation sind die Intertrigi- nes. Die Erkrankung kann sich aber auch in seltenen Fällen am Kapillitium, der Rumpfhaut oder der Mundschleimhaut manifestieren [3].

Histologisch sieht man eine suprabasale, akantholytische Spaltbildung mit epidermalen bzw. oberflächlich dermalen Entzündungsinfiltraten. Die Immunfluoreszenz entspricht i.W. der des Pemphigus vulgaris. Antikörper gegen Desmoglein und Periplakin sind nachweisbar [2].

Je nach Lokalisation der Erkrankung sind differenzialdiagnostisch andere Erkrankungen auszuschließen. Am Kapillitium muss auch an eine Trichophytie, eine vegetierende Pyodermie, eine Acne inversa oder an eine Folliculitis decalvans gedacht werden.

Die Therapie des Pemphigus vegetans entspricht der des Pemphigus vulgaris. Kortikosteroide als Monotherapie sind jedoch beim Pemphigus vegetans weniger erfolgreich als beim Pemphigus vulgaris. Deshalb empfiehlt sich eine Kombinationsbehandlung, z. B. mit Azathioprin $[4,5]$.

\section{Literatur}

1 Ahmed AR, Blose DA. Pemphigus vegetans. Neumann type and Hallopeau type. Int J Dermatol 1984; 23: 135 - 141

2 Cozzani E, Christana K, Mastrogiacomo A, Rampini P, Drosera M, Casu $M$, Murialdo G, Parodi A. Pemphigus vegetans Neumann type with anti-desmoglein anti-periplakin autoantibodies. Eur J Dermatol 2007; 17: $530-533$

3 Danapoulou I, Stavropoulos P, Stratigos A, Chatziolou E, Chiou A, Georgala S, Katsambas A. Pemphigus vegetans confined to the scalp. Int J Dermatol 2006; 45: 1008 - 1009

4 Jansen T, Messer G, Meurer M, Plewig G. Pemphigus vegetans. Hautarzt 2001; 52: 504-509

5 Kaiser J, Kaatz M, Elsner P, Ziemer M. Complete remission of drug-resistant Pemphigus vegetans treated by extracorporeal photopheresis. J Eur Acad Dermatol Venereol 2007; 21: $843-844$

\section{Schnitzler-Syndrom \\ $\nabla$ \\ H. Seifert, S. Großmann}

Seit Oktober 2005 leidet die 77-jährige Patientin unter einer rezidivierend auftretenden generalisierten Urtikaria mit mäßigem Juckreiz. Die Schübe wurden meist von Schüttelfrost und Fieber begleitet. Zudem berichtete die Patientin über starke brennende Knochenschmerzen der Schienbeine und Arthralgien in den Knie- und Ellenbogengelenken sowie einen Gewichtsverlust von ca. $10 \mathrm{~kg}$ im letzten $3 / 4$ Jahr und Appetitlosigkeit. Eine ambulant und stationär eingeleitete Diagnostik erbrachte bislang keine Aufschlüsse. Eine Prednisolontherapie führte nicht zur Besserung des Lokalbefundes.

Hautbefund: Es fanden sich generalisiert am gesamten Integument isoliert stehende runde makulopapulöse Urticae mit täglich wechselner Lokalisation ( $\bullet$ Abb. 9). Das Gesicht, die Kopfhaut, die Handflächen und Fußsohlen blieben ausgespart. Schwellungen im Sinne eines Quincke-Ödems wurden nicht beobachtet.

Laborbefunde: BSG 88 mm/h, CRP 124 mg/l, Erythrozyten 4 Tpt/l, $\mathrm{Hb} 6,7 \mathrm{mmol} / \mathrm{l}$, Leukozyten $18 \mathrm{Gpt} / \mathrm{l}$, Thrombozyten $700 \mathrm{Gpt} / \mathrm{l}$, Eiweißelektrophorese: Albumin 50,4\%, $\alpha 1$-Globulin 4,7\%, $\alpha 2$-Globulin: 10,8\%, $\gamma$-Globulin: 19,2\%, monoklonale IgM-Gammopathie Typ kappa im Serum, nicht jedoch im Urin nachweisbar.

Histologie: Epidermis überwiegend unauffällig, epidermale Basalmembran zart. Im Papillarkörper und oberen Korium zahl- 


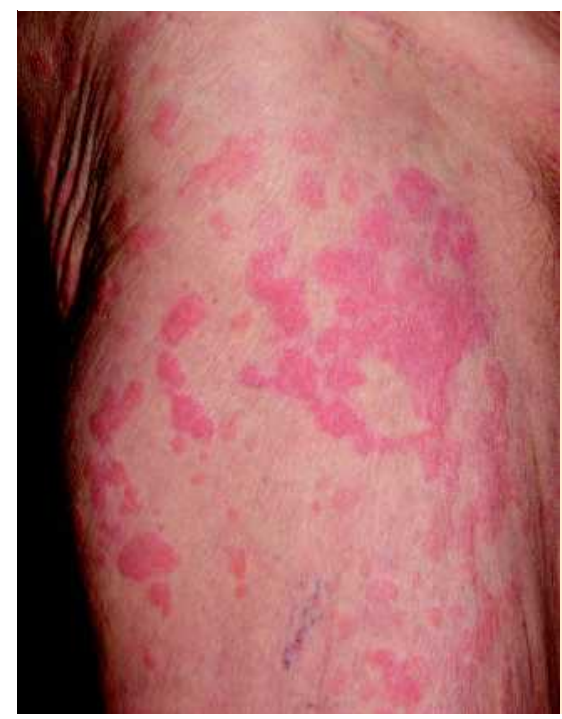

Abb. 9 Disseminierte Quaddeln bei Schnitzler-Syndrom.

reich nachweisbare Angiektasien und ein mitteldichtes, überwiegend perivaskulär lokalisiertes, aber auch interstitielles Infiltrat aus Lymphozyten, zahlreichen neutrophilen Granulozyten, einzelnen Eosinophilen sowie wenigen Mastzellen und monozytoiden Zellen. Teilweise Ausbreitung der entzündlichen Infiltrate bis in die mittlere Dermis. Keine fibrinoiden Gefäßwandnekrosen oder entzündliche Gefäßwandinfiltrate. Kein Nachweis spezifischer Immunreaktionen für IgA, IgM, IgG, C3, C4, C1q.

Bildgebende Diagnostik: In der Beckenübersicht: osteopenisch wirkende Knochenstrukturen, kein knochendestruierender Prozess. Gering bis mäßiggradig ausgeprägte Gonarthrose. In der Sonographie vergrößerte Lymphknoten inguinal. Mehrphasenknochenszintigraphie und Knie-SPECT: Vermehrter Knochenstoffwechsel vor allem in den Kniegelenken, aber auch im rechten Handgelenk-/Handwurzelbereich, im linken Daumensattelgelenk und in den Ileo-Sacral-Gelenken, die jedoch nicht typisch im Sinne von arthritischen Veränderungen sind.

Therapie und Verlauf: Antihistaminikatherapie mit Desloratadin (Aerius ${ }^{\circledR} 5$ mg 1-1-1) und Ranitidin (Ranibeta ${ }^{\circledR} 3001 / 2-0-1 / 2$ ), Lokaltherapie mit Betamethason-V-Creme 0,1\% NRF. Eine Indikation für Prednisolon bestand aufgrund des guten Ansprechens auf die o.g. Therapie zur Zeit nicht. Gegen die Knochenschmerzen wurde Etoricoxib (Arcoxia ${ }^{\circledR} 90 \mathrm{mg} / \mathrm{d}$ ) verordnet, worunter sich die subjektiven Beschwerden deutlich gebessert hatten.
Kommentar: Das Schnitzler-Syndrom ist eine seltene Erkrankung mit der Kombination aus chronischer Urtikaria, einer monoklonalen IgM-Gammopathie (Variante: IgG) und Arthralgien. Die Urticae sind bevorzugt am Stamm und den Extremitäten unter Aussparung des Gesichts, der Palmae und Plantae lokalisiert und im Verlauf von mildem Pruritus begleitet. Angioödeme werden selten beobachtet. Das mittlere Erkrankungsalter liegt bei 52 Jahren mit leichter Androtropie ( $\mathrm{M}: \mathrm{F}=1,6: 1)$ [1].

Rezidivierende Fieberschübe (90\% der Patienten) mit Temperaturen $>40^{\circ} \mathrm{C}$ und Gewichtsverlust werden regelmäßig beobachtet, ebenso Knochenschmerzen v.a. der Tibiae und der Ossa ilia (45\%) und Arthralgien (80\%), die v.a. die großen Gelenken wie Knie-, Hüft-, Hand- und Sprunggelenke betreffen. Zusätzlich können Lymphknotenschwellungen und eine Hepatosplenomegalie auftreten. Laborchemisch sind Anämien, Leukozytosen, Thrombozytosen und BSG-Erhöhungen typisch. Die Erkrankung verläuft chronisch. In ca. 15\% der Fälle entwickelt sich im Verlauf eine lymphoproliferative Erkrankung [1]. Differenzialdiagnostisch sollten lymphoproliferative Erkrankungen sowie ein adulter Mb. Still ausgeschlossen werden. Das Schnitzler-Syndrom ist ein Risikofaktor einer AA-Amyloidose.

Die Therapie des Schnitzler-Syndroms ist schwierig. Hochdosierte Glukokortikoide können eine vorübergehende Symptomreduktion erzielen. Antihistaminika sind in der Regel ebenso unwirksam wie Hydrochloroquin, Chloroquin, Dapson, Azathioprin, Pefloxazinmesylat oder Plasmapherese. Der IL-1-Rezeptorantagonist Anakinra führte in mehreren Fällen zur kompletten Remission [2, 3]. Zur Behandlung der intermittierenden Fieberschübe, der Knochenschmerzen und Arthralgien sind NSAIDs geeignet.

\section{Literatur}

1 de Koning HD, Bodar EJ, van der Meer JW, Simon A. Schnitzler Syndrome Study Group. Schnitzler syndrome: beyond the case reports: review and follow-up of 94 patients with an emphasis on prognosis and treatment. Semin Arthritis Rheum 2007; 37: 137-148

2 Ryan JG, de Koning HD, Beck LA, Booty MG, Kastner DL, Simon A. IL-1 blockade in Schnitzler syndrome: ex vivo findings correlate with clinical remission. J Allergy Clin Immunol 2008; 121: 260-262

3 Treudler R, Kauer F, Simon JC. Striking effect of the IL-1 receptor antagonist anakinra in chronic urticarial rash with polyclonal increase in IgA and IgG. Acta Derm Venereol 2007; 87: 280-281 